\title{
LA INTERVENCIÓN PEDAGÓGICA EN EDUCACIÓN FÍSICA
}

\section{PEDAGOGICAL INTERVENTION IN PHYSICAL EDUCATION}

\author{
Eulisis Smith Palacio ${ }^{1}$ \\ Jesús Alcalá Recuerdo² \\ Universidad Francisco de Vitoria. España
}

\section{RESUMEN}

El objetivo del presente estudio fue analizar las diferencias por curso en Educación Primaria Obligatoria, en la adquisición del autocontrol en preadolescentes que participaron del Programa de Intervención Educativa Delfos, Cecchini (2008), con un total de 204 alumnos de 6 colegios de Castilla La-Macha. Se utilizó el pos-test de las variables retroalimentación personal, retraso en la recompensa, autocontrol criterial, autocontrol procesual y sinceridad. El programa de intervención educativa consta de 20 sesiones de 1 hora de duración, en las clases de educación física. Se utilizó el cuestionario

1 Doctor en Investigación en Humanidades, Artes y Educación. Profesor del Grado de Ciencias de la Actividad Física y el Deporte. e.smith@ufv.es. Tel. 670582915. Universidad Francisco de Vitoria. España. https://orcid.org/00000001-9793-9476.

2 Doctor en Pedagogía de la Educación. Director del Máster de Profesorado. j.alcala@ufv.es. Tel. 661418798. Universidad Francisco de Vitoria. España. https://orcid. org/0000-0001-8496-5237
CACIA de Bonet, Moreno y Completo (1986), publicado por la editorial TEA Ediciones, S.A. Se confirma, una disminución significativa en cuanto a las protestas, la violación de las reglas del juego, al igual que la opinión sobre la búsqueda de la victoria a cualquier precio, resaltando valores prosociales, respeto a los compañeros y respeto a las normas de juego.

PALABRAS CLAVES: agresividad, deporte, actitudes, Programa Delfos

\section{ABSTRACT}

The objective of this study was to analyze the differences per course in Compulsory Primary Education,in the acquisition of self-control that pre-adolescents who preparticipated in the Delphi Educational Intervention Program, (Cecchini 2008), with a total of204 students from 6 schools in Castilla la Macha. The postest of the personal feedback variables, delay in reward, self-control criterial, processional self- 
control and sincerity was used. The educational intervention programme consists of 20,1 -hour sessions. The questionnaire CACIA de Bonet, Moreno y Completo (1986), published by the publisher TEA Ediciones, S.A. was used. It is confirmed, a significant decrease in protests, the violation of the rules of the game, as well as the opinion on the search for victory at any price, highlighting prosocial values, respect for the companions and respect for the rules of the game.

KEY WORDS: aggressiveness, sport, attitudes, Delphi Program

\section{INTRODUCCIÓN}

Naciones Unidas promueve la educación en valores a través de la UNESCO (2015), infancia y adolescencia UNICEF (2015), o salud OMS (2014). En este sentido incide notablemente en la edad evolutiva de la adolescencia, dada la importancia definitoria en la adquisición y consolidación de los valores educativos en la personalidad (González, Garcés de los Fayos y García, 2012). En este mismo sentido y con igual énfasis se plantea en el curriculum de Secundaria Básica y Bachillerato el desarrollo de actitudes y valores, integradores de conocimientos y habilidades transversales como el trabajo en equipo, el juego limpio o el respeto (Real Decreto 1105/2014).

La evidencia científica refleja con claridad, mediante autores como (Méndez-Giménez, Fernández-Rio, Cecchini y González de Mesa, 2013; Ntoumanis y Standage, 2009; Aguilar, Grau y Prat, 2015; Iturbide y Elosúa, 2012; Lamoneda, Huertas, Córdoba y García, 2015; Weinberg y Gould, 2014) las dos vías eficientes para el aprendizaje de valores, estas vías son: la práctica de la actividad deportiva y la educación física. Otros estudios, utilizan programas de intervención educativa con el fin de enseñar valores olímpicos en la sociedad como son: esfuerzo, compromiso, integración, participación, respecto, compañerismo (García, Arcas, Morote y Pato, 2018; Mosquera-González y Sánchez, 2015; Baena- Extremera; Granero-Gallegos, Martínez- Molina, 2015; Ortega, Jiménez, Giménez, Jiménez y Franco, 2012; Sukys y Majauskien, 2014).

El programa Delfos Cecchini (2008), es una intervención pedagógica mediante herramientas y principios educativos, con el fin de perseguir el desarrollo social de estudiantes en el marco de la Educación Física. Delfos, nace por la necesidad de validad un programa de intervención educativa para erradicar los comportamientos violentos de jóvenes perteneciente a los grupos ultras en los campos de fútbol. Este programa pretende, conseguir resultados similares lo encontramos en la Intervención psicoeducativa de García y Sánchez (2005), estos autores afirman que este tipo de intervención tiene cuatro pilares asociados ellos son: la prevención, la corrección optimización y compensación de aspectos psicológicos en el ámbito escolar (De Mendoza, Domínguez, Hernández, 2003). Pato et al. (2008); Oliva, Marcos y Ponce (2018), aseguran que se ha podido evidenciar un interés de las administraciones públicas para conseguir la integración de las nuevas generaciones a una sociedad competente y exigente de actitudes prosocial. Esta necesidad educativa se concreta en una meta básica, formar a ciudadanos capaces de vivir en una sociedad civilizada. El contexto de la Educación Física, puede ser un escenario idóneo para conseguir estas metas, así lo afirman (Berengüi y Garcés de los Fayos, 2007; Llamas y Cabrera, 2004; Madrona, et al., 2019; Gómez, 2005).

El objetivo del presente estudio fue analizar las diferencias por curso $5^{\circ}$ y $6^{\circ}$ de Educación Primaria Obligatoria, en la adquisición del autocontrol que tienen los adolescentes que participaron del Programa de Intervención Educativa Delfos, (Cecchini 2008) en las variables retroalimentación personal, retraso en la recompensa, autocontrol 
criterial, autocontrol procesual y sinceridad. Se analizó si: ¿se producen cambios significativos en la adquisición del autocontrol? ¿si la adquisición de los valores afecta a todos los alumnos por igual, independientemente de su edad? ¿cómo afecta esta adquisición de valores en la familia y el colegio?

\section{MÉTODO}

\section{PARTICIPANTES}

Los participantes fueron un total de 204 estudiantes, 92 chicas $(54,1 \%)$ y 78 chicos $(45,8$ $\%)$ con unas edades que oscilan entre 11 y 13 años ( $M$ edad= 11, 7; DT=0,61) y que cursaban Educación Física en quinto y sexto curso de Educación Primaria en 6 centros de Castilla-La Mancha. La facilidad de acceso al centro fue determinante en la elección de la muestra.

\section{INSTRUMENTO}

Se usó el cuestionario CACIA de Bonet, Moreno y Completo (1986), publicado por la editorial TEA Ediciones, S.A. con las escalas de Retroalimentación Personal, Retraso en la Recompensa, Autocontrol Criterial, Autocontrol Procesual y Sinceridad. El cuestionario CACIA, es un cuestionario de autocontrol infantil, para niños entre 11 y 19 años. Desde $5^{\circ}$ de primaria a $2^{\circ}$ de Bachiller. Sus escalas se centran en la evaluación del autocontrol considerado desde el punto de vista conductual. Cuya base es precisamente el esfuerzo de las personas por cambiar su actitud. También mide el esfuerzo de las personas por modificar sus reacciones. $Y$ esta modificación de las reacciones son necesarias en el fútbol o en cualquier otro Deporte, que requiera autocontrol.

Además, el cuestionario permite medir cinco escalas: Retroalimentación personal, Autocontrol criterial, Retraso en la recompensa, Autocontrol procesual y Sinceridad. De las cinco escalas tres miden aspectos positivos (RP) (RR) (ACC), una mide aspectos negativos (ACP) y la última mide nivel sinceridad(S) nos indica espontaneidad, impulsividad, independencia de normas y exigencia sociales. Retroalimentación personal: está compuesta por 21 elementos. Una puntuación elevada indica la capacidad para conocerse a sí mismo/a, darse cuenta de sus actos propios, el interés por los motivos y razones que te hacen actuar de una manera determinada. (Cuando me dicen que debo portarme bien suelo preguntar. “¿Qué quiere decir eso?”, ¿A veces me dicen que soy incapaz de saber lo que está bien o mal hecho). Retraso de la recompensa: las conforman 19 elementos, recoge la regulación sobre respuestas impulsivas, te permite dar prioridad a lo urgente y a lo importante, dejando a un lado lo que te apetece. Por tanto, las puntuaciones altas nos indican un alto nivel de organización en el trabajo y en las acciones. De forma general una persona con retraso en la recompensa no se dejaría llevar por los actos de impulsos. (<Recuerdo mis obligaciones en casa>, < Muchas veces evito hacer algo que no me gusta porque sé que no debo>). Autocontrol criterial: está compuesta por 10 elementos, refleja la capacidad de las personas para soportar situaciones dolorosas o desagradables, también recoge aspectos de la responsabilidad personal. Sujetos con una puntuación elevada tendría una buena resistencia al estrés y situaciones amenazantes, mostrándose responsables, donde otras personas se asustarían. (<Soy capaz de aguantar que me peguen $\mathrm{o}$ insulten con tal de hacer algo que creo que debo hacer $>$, $<$ Si tengo miedo por algo me aguanto y hago lo que tengo que hacer>). Autocontrol procesual: La forman 25 aspectos, conformado principalmente por autoevaluación, autogratificación, autocastigo. Una alta puntuación significaría que existe mucha preocupación por siempre actuar bien, un gran miedo al fracaso, o un gran sentimiento al rechazo. También esta escala nos indica el grado de autoestima que tiene la persona sobre sí misma, ansiedad por el logro de la meta, una supervaloración de los objetivos. (<Cuando cometo errores me critico a mí mismo $>,<$ Cuanto 
más consigo por encima de lo que me había propuesto y sé que se debe a mi propio esfuerzo, más a gusto me siento>). Escala de sinceridad: la componen 14 elementos, una baja puntuación en esta escala supondría una alta dependencia a las normas sociales, y una lata puntuación refleja espontaneidad, impulsividad, independencia de las normas.

Para el estudio de la consistencia interna de este cuestionario fue utilizado el coeficiente alfa de Cronbach, para la escala de retroalimentación personal=.79; el autocontrol procesual=.76; el retraso en la recompensa $=.71$; el autocontrol criterial $=.50$ y la sinceridad $=.63$.

\section{PROCEDIMIENTO}

Se dividieron los grupos, en grupo experimental y grupo de control, a todos los grupos se le aplicó el cuestionario CACIA. A partir de este momento y durante 10 semanas (20 sesiones de una hora de duración) al grupo experimental se le aplicó el programa Delfos por profesores formados a tal efecto. El programa tenía como objetivo disminuir las conductas agresivas y mejorar el razonamiento en las clases de Educación Física diarias y por tanto se esperaba que se eliminaran las conductas violentas. En el mismo periodo de tiempo, el grupo de control realizó las clases de Educación Física que estaban programas, pero sin aplicar el programa Delfos. Con el fin de formar a los profesores, se desarrollaron tres seminarios y sesiones modelos.

\section{DISEÑO Y VALIDACIÓN DEL PROGRAMA DE INTERVENCIÓN}

En primer lugar, el investigador diseñó el programa de intervención educativa con un total de 20 sesiones, siguiendo la estructura del programa Delfos (Cecchini, Losa, González Arruza, 2008), para el aprendizaje de fútbol. El programa consistió en una unidad didáctica de fútbol con una extensión de 20 sesiones, de 55 minutos cada una, estas sesiones se impartían dos veces por semana a lo largo de dos meses y medio. Se consideró suficiente las 20 sesiones para mejorar el autocontrol, basado en los trabajos de (Lamoneda, Huertas, Córdova y García, 2015; De Mendoza, Medina y Hernández. 2003). El programa se realizó dentro del horario que los colegios tienen reservados para la Educación Física.

Se realizaron 6 formaciones con todos los profesores sobre el programa Delfos, donde se analizaron las estrategias educativas y los principios pedagógicos del programa (Cecchini, Cecchini, Fernández y González, 2011). Se realizaron tres sesiones de exposición de la metodología Delfos explicando al detalle cada momento de la sesión. El programa fue validado por tres expertos en este ámbito y se procedió a la presentación a un comité de Ética para su revisión. Los profesores encargados de aplicar el programa Delfos en los colegios, tenían entre 7 y 20 años de experiencia, todos recibieron la formación necesaria para aplicar el programa

Se tuvieron en cuenta en las dos sesiones iniciales, la explicación del programa a los alumnos y a partir de aquí cada tres sesiones tenían un objetivo para desarrollar el autocontrol y el jugo limpio. Los objetivos eran: el respeto a los compañeros de equipo, no buscar la vitoria a cualquier precio, respeto a los árbitros, respeto a los adversarios y no perder tiempo para obtener la victoria. Junto con estos objetivos, al final de cada sesión se analizaba un dilema moral en relación con el objetivo que se estaba trabajando, los alumnos levantaban la mano y participaban dado su opinión sobre el dilema moral y escuchando la exposición de otros compañeros. Cada una de las sesiones, se iniciaba con el análisis entre el profesor y el alumno del objetivo de la sesión, en segundo lugar se realizaba un calentamiento o activación, en tercer lugar se realizaron varios ejercicio de confrontación donde los alumnos jugaban con superioridad numérica, con algún tipo de desventaja como jugar con la pierna débil 
o con limitación de la zonas, en cuarto lugar, se analizaba un dilema moral que están relacionado con el objetivo de la sesión y en quito lugar se enviaba un folio para que los alumnos redactaran alguna experiencia parecida al dilema moral en la familia o en el colegio. El momento de los dilemas morales, duraba 10 minutos, se les pedía a los alumnos que respetaran lo criterios de otros y que esperan su turno de palabra, un ejemplo de dilema moral es el siguiente: Un jugador de mi equipo insulta al jugador del equipo contrario, para ganar tiempo. Los insultos que usa son bastantes duros para además buscar la desconcentración de este jugador. Mi equipo va perdiendo $4 \times 3$. El entrenador para este partido, nos ha dicho que hay que ganar de cualquier manera. ¿Cuál es tu opinión sobre este caso? ¿Cómo actuaría si fuera tu compañero de equipo?

\section{DISEÑO Y VALIDACIÓN DEL PROGRAMA DE INTERVENCIÓN}

En primer lugar, el investigador diseñó el programa de intervención educativa con un total de 20 sesiones, siguiendo la estructura del programa Delfos (Cecchini, Losa, González Arruza, 2008), para el aprendizaje de fútbol. El programa consistió en una unidad didáctica de fútbol con una extensión de 20 sesiones, de 55 minutos cada una, estas sesiones se impartían dos veces por semana a lo largo de dos meses y medio. Se consideró suficiente las 20 sesiones para mejorar el autocontrol, basado en los trabajos de (Lamoneda, Huertas, Córdova y García, 2015; De Mendoza, Medina y Hernández. 2003). El programa se realizó dentro del horario que los colegios tienen reservados para la Educación Física.

Se realizaron 6 formaciones con todos los profesores sobre el programa Delfos, donde se analizaron las estrategias educativas y los principios pedagógicos del programa (Cecchini, Cecchini, Fernández y González, 2011). Se realizaron tres sesiones de exposición de la metodología Delfos explicando al detalle cada momento de la sesión. El programa fue validado por tres expertos en este ámbito y se procedió a la presentación a un comité de Ética para su revisión. Los profesores encargados de aplicar el programa Delfos en los colegios, tenían entre 7 y 20 años de experiencia, todos recibieron la formación necesaria para aplicar el programa

Se tuvieron en cuenta en las dos sesiones iniciales, la explicación del programa a los alumnos y a partir de aquí cada tres sesiones tenían un objetivo para desarrollar el autocontrol y el jugo limpio. Los objetivos eran: el respeto a los compañeros de equipo, no buscar la vitoria a cualquier precio, respeto a los árbitros, respeto a los adversarios y no perder tiempo para obtener la victoria. Junto con estos objetivos, al final de cada sesión se analizaba un dilema moral en relación con el objetivo que se estaba trabajando, los alumnos levantaban la mano y participaban dado su opinión sobre el dilema moral y escuchando la exposición de otros compañeros. Cada una de las sesiones, se iniciaba con el análisis entre el profesor y el alumno del objetivo de la sesión, en segundo lugar se realizaba un calentamiento o activación, en tercer lugar se realizaron varios ejercicio de confrontación donde los alumnos jugaban con superioridad numérica, con algún tipo de desventaja como jugar con la pierna débil o con limitación de la zonas, en cuarto lugar, se analizaba un dilema moral que están relacionado con el objetivo de la sesión y en quito lugar se enviaba un folio para que los alumnos redactaran alguna experiencia parecida al dilema moral en la familia o en el colegio. El momento de los dilemas morales, duraba 10 minutos, se les pedía a los alumnos que respetaran lo criterios de otros y que esperan su turno de palabra, un ejemplo de dilema moral es el siguiente: Un jugador de mi equipo insulta al jugador del equipo contrario, para ganar tiempo. Los insultos que usa son bastantes duros para además buscar la desconcentración de este jugador. Mi equipo va perdiendo $4 \times 3$. EI entrenador para este partido, nos ha dicho que hay que ganar de cualquier manera. ¿Cuál es 
tu opinión sobre este caso? ¿Cómo actuaría si fuera tu compañero de equipo?

\section{ANÁLISIS DE LOS DATOS}

Se utilizó el programa estadístico IBM-SPSS 19.0 para el tratamiento de los datos. Se comprobó la distribución de los valores de las variables mediante las pruebas de KolmogorovSmirnov, Shapiro- Wilks, Asimetría y Curtosis para comprobar la normalidad de la muestra obteniendo unos valores de $p>0,05$. Se cumple el principio de normalidad en las variables: autocontrol criterial, autocontrol procesual, retroalimentación personal, se realizó para estos casos un análisis de varianza simple. No se cumple el principio de normalidad en las variables: retraso en la recompensa y sinceridad, se realizó la prueba de "Chi" Cuadrado. Se calculó las medias y la desviación típica para las dos mediciones pos test, para determinar la diferencia entre los alumnos de $5^{\circ}$ y $6^{\circ}$ curso de la Educación Primaria. En ambos casos se tomó como referencia el pos- test. Los datos obtenidos son el resultado de la aplicación del cuestionario CACIA.

Para poder llevar a cabo el análisis que nos conducirá a la verificación de la hipótesis planteada, hemos transformado los resultados obtenidos de la aplicación de la escala. La escala utilizada da valores Sí y No, que son propios de una variable con nivel de medición nominal. Las variables con nivel de medición nominal solo permiten la realización de análisis no paramétricos lo que limita la posibilidad de generalización de los resultados a la población de la que se ha extraído la muestra; además, estos análisis tienen una potencia menor que los análisis realizados con pruebas paramétricas, es decir, hay mayor riesgo de aceptar hipótesis nulas falsas. Por tanto, en nuestra opinión, es conveniente, siempre que sea posible, utilizar para el análisis pruebas paramétricas, valorando en todo caso que estas pruebas exigen el cumplimiento de una serie de supuestos (independencia, homogeneidad de varianzas, normalidad, fundamentalmente).

Para poder aplicar pruebas paramétricas, se procedió a transformar los resultados de la aplicación de la escala, asignando un valor 1 a la respuesta sí y un valor 0 a la respuesta no. Se decidió hacerlo así para reflejar mejor las diferencias en las respuestas en el pre-test y el pos-test: un aumento en la media de las puntuaciones de la variable en el pos-test con respecto al pre-test, nos estaría indicando un efecto positivo del programa de intervención educativa.

Hay que tener en cuenta que dos de las escalas medidas - "Autocontrol Procesual" y "Sinceridad" - tienen una orientación negativa, es decir, la obtención de puntuaciones altas en estas escalas indica un resultado negativo. En estas dos escalas se ha sustituido la respuesta "si" por el valor 0 y la respuesta "no" por el valor 1 .

\section{RESULTADOS}

\section{CONTRASTE DE MUESTRAS RELACIONADAS}

En la Tabla 1, podemos observar el contraste de muestras relacionadas, la cual permite comparar las medias y la significatividad de las diferencias entre las medias de todas las variables. Esta prueba es importante, dado que ayuda a comparar de forma directa los resultados del pretest y pos-test y obtener una valoración concreta. 


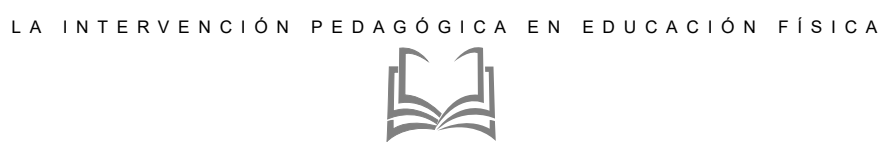

Tabla 1. De Diferencias Relacionadas

\begin{tabular}{|c|c|c|c|c|c|c|c|c|}
\hline & \multicolumn{5}{|c|}{ Diferencias relacionadas } & \multirow[t]{3}{*}{$t$} & \multirow[t]{3}{*}{$g l$} & \multirow{3}{*}{$\begin{array}{l}\text { Sig. } \\
\text { (bilateral) }\end{array}$} \\
\hline & Media & $\begin{array}{l}\text { Desviación } \\
\text { típ. }\end{array}$ & $\begin{array}{l}\text { Error } \\
\text { típ. } \\
\text { de la }\end{array}$ & \multicolumn{2}{|c|}{$\begin{array}{l}95 \% \text { Intervalo de } \\
\text { confianza para la } \\
\text { diferencia }\end{array}$} & & & \\
\hline & & & media & Inferior & Superior & & & \\
\hline $\begin{array}{l}\text { Retroal. Personal } \\
\text { pretes suma - Retroal. } \\
\text { Personal postest suma }\end{array}$ & 2,60 & 3,36 & 0,24 & 2,13 & 3,07 & 10,9 & 199 & 0,00 \\
\hline $\begin{array}{l}\text { Retraso Recomp } \\
\text { pretest - Retraso }\end{array}$ & 0,75 & 3,00 & 0,21 & 0,34 & 1,17 & 3,59 & 203 & 0,00 \\
\hline Recomp postest & & & & & & & & \\
\hline $\begin{array}{l}\text { Autocont Criterial } \\
\text { pretest - Autocont } \\
\text { Criterial postest }\end{array}$ & $-0,64$ & 2,07 & 0,14 & $-0,93$ & $-0,36$ & 4,46 & 203 & 0,00 \\
\hline $\begin{array}{l}\text { Autocont Procesal } \\
\text { pretest - Autocont } \\
\text { Procesal postest }\end{array}$ & 2,13 & 3,41 & 0,24 & 1,66 & 2,61 & 8,84 & 198 & 0,00 \\
\hline Sinceridadsumapretest & & & & & & & & \\
\hline- & 0,16 & 2,18 & 0,15 & $-0,14$ & 0,46 & 1,06 & 203 & 0,29 \\
\hline
\end{tabular}

Para mayor profundización en los resultados, nos sumergiremos en la búsqueda de datos que muestren, las diferencias entre las variables y la intensidad de esta correlación. De forma general podemos decir que existen cambios significativos en las variables Retroalimentación Personal (la autoobservación, autoconcepto) $\quad(\mathrm{t}=10.9)$; ( $p<0.00)$; $(D T=3.36)$, Retraso en la Recompensa (control de las actitudes impulsivas) ( $\mathrm{t}=3.59)$; $(p<0.00) ; \quad(D T=3.00), \quad$ Autocontrol Criterial (capacidad de soportar situaciones difíciles y la autoefiCACIA) ( $\mathrm{t}=4.46) ; \quad(p<0.00) ; \quad(\mathrm{DT}=2.07)$, Autocontrol Procesual (la autovaloración y la persistencia) $\quad(t=8.84) ; \quad(p<0.00) ; \quad(D T=3.41)$, no siendo así en la variable Sinceridad (la espontaneidad) ( $t=1.06)$; $(p>0.29)$; (DT=2.18).

\section{ANÁLISIS DE VARIANZA SIMPLE AUTOCONTROL CRITERIAL, AUTOCONTROL PROCESUAL Y SINCERIDAD}

En la variable Autocontrol Criterial que es la capacidad de un individuo para soportar situaciones desagradables y la sensación de autoefiCACIA, se obtuvo una media pos-test para $5^{\circ}$ de primaria de 6,20 y para $6^{\circ}$ de primaria 6,23 , estos resultados indican la capacidad de los alumnos para soportar situaciones desagradables y de responsabilidad personal. Podemos observar que no existen diferencias significativas entre estos grupos de alumnos ( $F=0,01 ; p>0,94)$ entre los chicos de $5^{\circ}$ y $6^{\circ}$ de primaria en el soportar situaciones difíciles y desagradables.

En el caso de la variable negativa Autocontrol Procesual que significa la capacidad de autovaloración de un individuo y el nivel de persistencia, se alcanzó una media de 4,74 para $5^{\circ}$ de primaria y 5,02 para $6^{\circ}$ de primaria, como podemos ver los valores medios son muy parecidos, siendo la diferencia estadísticamente no significativa ( $F=0,27 ; p>0,61)$, esto indica que se autovaloran de la misma manera los chicos de $5^{\circ}$ y de $6^{\circ}$ de primaria que han participado en la investigación. 


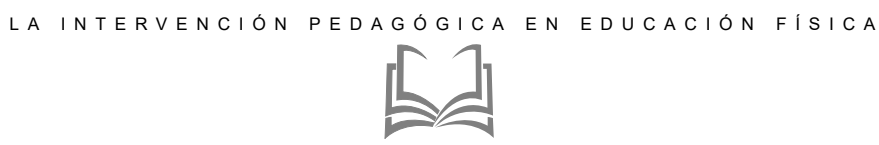

En la variable Sinceridad que es la capacidad de los alumnos para actuar de forma espontánea se obtuvo una media de 7,02 para $5^{\circ}$ de primaria y 6,89 para $6^{\circ}$ de primaria, obteniendo unos resultados estadísticamente no significativos $(F=0,13 ; p>0,72)$, esto indica que el programa no ha tenido influencia en la mejora de la Sinceridad y la espontaneidad de estos alumnos.

Podemos concluir diciendo que no existen cambios significativos por curso en cuanto a las variables Autocontrol Criterial, Autocontrol Procesual y Sinceridad en la aplicación del programa Delfos en los niños que han participado en la investigación.

Tabla 2. Análisis de varianza simple Autocontrol Criterial, Autocontrol Procesual y Sinceridad por curso

\begin{tabular}{llll}
\hline \hline \multicolumn{1}{c}{ Análisis por curso escolar } & Medias & F & Sig. \\
& $5^{\circ}-6^{\circ}$ & & \\
Autocontrol Criterial & $6,20-6,23$ & 0,01 & 0,94 \\
Autocontrol Procesual & $4,74-5,00$ & 0,27 & 0,61 \\
Sinceridad & $7,02-6,89$ & 0,13 & 0,72 \\
\hline
\end{tabular}

\section{PRUEBA DE CHI CUADRADO PARA LAS VARIABLES RETRASO EN LA RECOMPENSA Y RETROALIMENTACIÓN PERSONAL}

Se han contrastado a continuación las diferencias del pos test entre las variables Retroalimentación Personal, que está relacionada con la autoobservación- autoconcepto y el análisis de situaciones y el Retraso en la Recompensa relacionado con el control de las respuestas impulsivas, teniendo en cuenta el curso $\left(5^{\circ} \circ 6^{\circ}\right.$ de Primaria).

En la variable Retroalimentación Personal que indica la capacidad de autoobservación y análisis de situaciones, se alcanzó una media para $5^{\circ}$ de primaria de 6,93 para $5^{\circ}$ de primaria y de 7,55 para $6^{\circ}$ de primaria, obteniendo unos resultados estadísticamente no significativos ( $\mathrm{x} 2=0,51$; $p>0,47$ ), los resultados advierten que el nivel de autoobservación y de autoconcepto entre los alumnos de $5^{\circ}$ y $6^{\circ}$ de primaria son iguales.

Así mismo en las variables Retraso en la Recompensa que indica el control de las actitudes impulsivas, arrojó una media 8,87 para $5^{\circ}$ de primaria y 9,61 para $6^{\circ}$ de primaria, siendo unos resultados estadísticamente no significativos $(x 2=2,71 ; p>0,09)$, esto advierte que los niveles de control de las actitudes impulsivas son iguales en los niños de $5^{\circ}$ y $6^{\circ}$ curso de primaria.

Podemos concluir diciendo, que los niveles de autoobservación y control de las actitudes agresivas son iguales en los alumnos de $5^{\circ}$ y $6^{\circ}$ de primaria, que han participado en el programa de intervención educativa Delfos Tabla 18.

Tabla 3. Análisis Retroalimentación Personal, Retraso en la Recompensa por cursos

\begin{tabular}{lll}
\hline \hline & Retroalimentación Personal & Retraso Recompensa \\
Chi-cuadrado & 0,51 & 2,71 \\
gl & 1 & 1 \\
Sig. asintót. & 0,47 & 0,09 \\
Medias $5^{\circ}-6^{\circ}$ & $6,93-7,55$ & $8,87-9,61$ \\
\hline \hline
\end{tabular}


Se concluyó que no existen diferencias significativas en ninguna de las dos variables (Retroalimentación Personal y Retraso en la Recompensa), ya que el valor de probabilidad de los resultados obtenidos es superior a 0,05. Por tanto, podemos decir que no existen diferencias significativas por curso.

\section{DISCUSIÓN}

Se confirma, mediante este estudio la efiCACIA del programa Delfos de Cecchini (2008), para generar cambios en cuanto a la creencia y opiniones, relacionada con el autocontrol de los chicos y chicas que han participado en este estudio. Se confirma una disminución significativa en cuanto a las protestas, la violación de las reglas del juego, al igual que la opinión sobre la búsqueda de la victoria a cualquier precio, resaltando valores de proactividad, respeto a los compañeros y respecto a las normas de juego. En concordancia con nuestro estudio podemos encontrar, un programa de Pensamiento Prosocial en Entornos Educativos y dirigido a alumnos con riesgo de inadaptación social de Educación Secundaria, concluye con mejora en la retroalimentación personal y mejora en el retraso en la recompensa (Fernández, Viguer y Cantero, 2015). En esta misma línea, una investigación relacionada con la adquisición de valores entre los chicos y las chicas a través del deporte, confirma que existe una predisposición independiente del sexo por los valores de esfuerzo, respeto y compañerismo, siendo por otro lado situados los valores de libertad, igualdad y cooperación relegados a un segundo plano (Martínez, et al., 2014).

Este modelo de programas de intervención educativa se basa en que los valores aprendidos en los espacios deportivos sean transferidos hacia otros entornos como es la familia y las aulas, con el diseño especifico de sesiones para este fin (Danish, Pepitas y Hale, 1990). El resultado hallado en esta investigación se relaciona con la aplicación del programa Delfos, implementado por Cecchini, Cecchini, Fernández y González (2011). Estos autores compararon las actitudes agresivas, actitudes competitivas y las actitudes cooperativas en las clases de educación física. En este sentido, constataron una disminución de las actitudes agresivas, tanto las reactiva como instrumentales. Este hallazgo también coincide con Cecchini, Fernández, González y Arruza, (2008), en el que encontraron una mejora significativa en las escalas de retroalimentación personal, retraso en la recompensa, autocontrol criterial y autocontrol procesual en los estudiantes que participaban de la investigación.

Se pudo comprobar en esta investigación, que en la medida que trascurrían las sesiones los alumnos ganaban confianza en las explicaciones, por ejemplo: en la sesión 12 donde el objetivo era respecta la normar del juego y no ganar ventaja sobre el contrario a la cualquier costa, en esta sesión los alumnos comprendieron lo injusto que es buscar ganar la ventaja haciendo acciones ilegales como atarse los cordones para perder tiempo o patear el balón lejos de la zona de saque para demorar el mismo. Este debate entre los alumnos y profesores se realizó mediante el siguiente dilema moral: -un jugador del equipo adversario te golpea de forma constante. Después de reclamarle al árbitro varias veces decides, atacar a este jugador y te envuelve con él en una pelea. ¿Ha sido correcta tu actitud? ¿Cómo actuarias en esta situación? -. Al finalizar cada sesión y a para asegurarnos que existía transferencia de los valores aprendidos a la familia o el colegio se solicitó a los alumnos una hoja de autoevaluación donde describiera alguna situación parecida en la familia o en casa.

Queda constatado de esta manera, que no es la dimensión práctica del deporte la que, sin más, garantiza la conquista de los valores en el deporte, sino el contexto educativo en el que se desarrolla el deporte. Tenemos que deshacer la idea, de que el deporte por si mismo, mejora el trabajo en equipo, el compañerismo, el esfuerzo, 
la solidaridad, el respeto a los compañeros, el respeto a las normas de juego y el respeto a la figura de referencia como pueden ser los árbitros, profesores, entrenadores y monitores. Tampoco se puede afirmar, que la simple práctica deportiva permita transferir los valores al entorno de la familia y el colegio, sino que se necesita implementar herramientas educativas diseñadas con este fin. El campo relacional e interactivo que permite la práctica deportiva, puede ser ideal para adquisición de valores, ejercerá una influencia decisiva los padres, entrenadores y en última instancia los propios deportistas con el fin de que el deporte sea un espacio de desarrollo moral y de razonamiento.

\section{CONCLUSIONES}

Podemos concluir diciendo que en esta aplicación del programa Delfos, en los 6 colegios donde se ha realizado la intervención educativa, no se han encontrado diferencias entre los alumnos de $5^{\circ}$ y $6^{\circ}$ curso, en la adquisición del autocontrol mediante las clases de educación física. La aplicación de programa Delfos, que trata sobre eliminar actitudes agresivas y mejorar el juego limpio, se advierte que se producen mejoras en los niveles de desarrollo personal y liderazgo. La aplicación del programa Delfos benefician los niveles de Autocontrol Criterial, el Autocontrol Procesual mediante este tipo de intervenciones educativas. Los niveles de Retroalimentación Personal y Retraso en la Recompensa mejoran si se diseñan herramientas educativas como el programa Delfos. Encontramos mejoría en el proceso de autoconocimiento personal, debido al aumento de la variable Retroalimentación Personal mediante la aplicación del programa Delfos. Por este hecho podemos decir que, la Educación Física y el Deporte pueden mejorar el carácter en los alumnos, si se aplican herramientas educativas con este fin. La capacidad de aceptar situaciones adversas y responder a ellas de forma adecuada, se pueden conseguir aplicando herramientas educativas de este tipo. Hoy en día se hace necesario, buscar una transformación personal que venga desde dentro. Estos cambios se pueden experimentar mediante la aplicación de programas educativos como él se ha utilizado en nuestro estudio.

\section{REFERENCIAS BIBLIOGRAFICAS}

Aguilar, G. F., Grau, M. P., y Prat, S. S. (2015). La intervención pedagógica del profesorado de educación física en un contexto multicultural: prácticas, reflexiones y orientaciones. Retos: Nuevas Tendencias en Educación Física, Deportes y Recreación, (28), 248-255.

Baena- Extremera, A., Granero-Gallegos, A., y Martínez- Molina, M (2015). Validación española de la escala de Evaluación de la Competencia Docente en Educación Física de Secundaria. Cuaderno de Psicología del Deporte, 15 (3), 113122.

Berengüí Gil, R., y Garcés de los Fayos, Ruiz, E. J. (2007). Los valores en el deporte escolar, estudio con profesores de educación física. Cuaderno de Psicología del Deporte, 7(2),90103.

Cecchini, J. A., Losa, J. F., González, C., y Arruza, J. A. (2008). Repercusiones del Programa Delfos de educación en valores a través del deporte en jóvenes escolares. Effect of the Delfos Program of Education in Values Thrugh Sport in Studentes. Revista Educación, 346, 167-186.

Cecchini, C., Cecchini, J., A., Fernández, J., L. y González, C. (2011). Repercusión del programa Delfos sobre los niveles de agresividad en las clases de educación física: actividades colaborativas versus competitivas. Magister: Revistas Miscelánea de Investigación, (24), 1121.

Danish, S.J., Pepitpas, AJ., y Hale,B. D. (1990). Sport as a context for developing competence. Developing Social Competency in Adolescence 3,169-194. 
De Mendoza, R. A., Medina, R. D., y Hernández, P, H. (2003). Evaluación de la mejora en valores de realización personal y social en adolescentes que han participado en un programa de intervención. Psicothema, 15(4), 589-594.

Fernández, M., A., Viguer, P., y Cantero, M. J. (2015). EfiCACIA del Pensamiento Prosocial en Entonos Educativos para el desarrollo de la competencia social de adolescentes en situación de riesgo de inadaptación social. Información Psicológica, 110, 39-50

García, J. L. J., Arcas, A. L., Morote, J. M., y Pato, A. S. (2018). Proyecto educativo Olimpízate: objetivos, metodologías y actividades para enseñar Olimpismo en los centros de Educación Secundaria. Retos: Nuevas Tendencias en Educación Física, Deporte y Recreación, (33), 286-292.

García, J., A., y Sánchez, J. M. R. (2005). Prácticas educativas familiares $\mathrm{y}$ autoestima. Psicothema, 17(1), 76-82.

Gil-Madrona, P., Gutiérrez-Marín, E. C., Cupani, M., Samalot-Rivera, A., Díaz- Suárez, A., y López- Sánchez, G. F. (2019). The effects of an appropiate behavior programo an elementary school children social skills development in physical education. Frontiers in psychology, 10.

González, J., Garcés de los Fayos, E., y García, A. (2012). Indicadores de bienestar psicológico percibido en alumnos de educación Física. Revista Psicología del deporte, 21(1), 183-187.

Gómez Rijo, A. (2005). La enseñanza y el aprendizaje de los valores en la educación deportiva. Revista internacional de Medicina y Ciencias de la Actividad Física y del Deportes/ Internacional Journal of Medicine and Science of Physical Activity and Sport, 5 (18), 89-99-
Iturbide Luquin, L. M., y Elosua Oliden, P. (2012). Perception of fair play in children and youth sport. Revista de Psicología del Deporte, 21(2), 253259.

Lamoneda Prieto, J., Huertas Delgado, F. J., Córdoba Caro, L. G., y García Preciado, A. V. (2015). Desarrollo de los componentes sociales de la deportividad en futbolistas alevines. Cuaderno de Psicología del Deporte, 15(2), 113124.

Martínez, R., Cepero, M., Collado, D., Padial, R., Pérez, A., y Palomares, J. (2014). Adquisición de valores y actitudes mediante el juego y el deporte en educación física, en educación secundaria. Journal of Sport \& Health Research, 6(3), 207216.

Meroño, L., Bada, J., Sánchez-Pato, A., Calderón, A., y Figuer-Montero, C. (2016). Más rápido, más alto, más fuerte: percepción del alumnado de Educación Secundaria sobre sus actitudes y comportamientos a través del aprendizaje Basado en Problemas. Revista Italiana di Pedagogía dello Sport, 1, 13-28.

Méndez- Giménez, A., Fernández- Rio, J., Cecchini, J. A., y González de Mesa, C. (2013). Perfiles motivacionales y sus consecuencias en educación Física. Un estudio complementario de metas de logro $2 \times 2$ y autodeterminación. Revista Psicología del Deporte, 22(1), 0029-38.

Mosquera- González M. J., \& Sánchez A. (2015). Modéle éducatif de la $<<$ non violence $>>$ et du fairplay pour le sport et pour la vie. Les régles éducatives á Travers le Code de la $<<$ nonviolence>>et du fair-play. Movement \& Sport Science y Motricé. Doi: 10.1050/sm/2015016

Ntoumanis, N., \& Standage, M. (2009). Motivation in phisical education classes: A self-determination theory perspective. Theory and Reasearch in Education, 7(2), 194-202. 


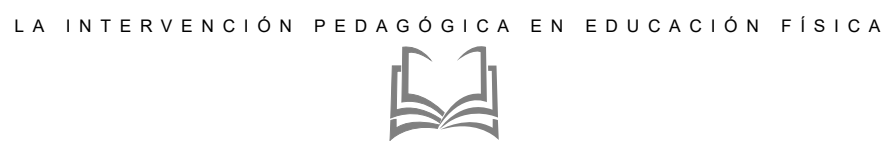

OMS. (2014). Informe sobre la situación mundial de la prevención de la violencia 2014. Organización Mundial de la Salud. Recuperado: https://www.who.int/violence_injury_prevention/ violence/status_report/2014/es/

Ortega, G., Jiménez, A., Giménez, J., Jiménez, P., y Franco, J. (2012). Utilización de cuadernos didácticos para educar en valores: un reto de las escuelas de Baloncesto de la Fundación Real Madrid. Cuaderno de Psicología del deporte, 12, 41-44.

Oliva, D. S., Marcos, F. M. L., y Ponce, I. G. (2018). Desarrollo de valores a través de las clases de educación física: una aproximación teórico-práctica. Perspectivas en la Psicología del Deporte: Investigación Aplicada, 59.

Pato, S., A, Bada, J., Juan de Dios, A. Mosquera, M., J., y Sánchez, C., Y. (2008). Educación en Valores a través del deporte. Sevilla, España: Wanceulen Editorial deportiva.

Real Decreto 1105/2014, de 26 de diciembre, por el que se establece el currículo básico de la Educación Secundaria Obligatoria y de Bachillerato (BOE 3, de sábado 3 de enero de 2015), 169.

Ruiz, J., y Cabrera, D. (2004). Los valores en el deporte. Revista de Educación, 335, 9-19.

Sukys, S., \& Majauskien, D. (2014). Effects o fan integrated Olympic educatión programo on adolecent athetes values and sport behavior. Social Behavior and Personality: an internacional journal, 42(5), 811-821.

UNESCO (2015). La educación para todos, 200-2015: Logros y desafíos. Organización de las Naciones Unidas para la Educación, La Ciencia y la Cultura. Recuperado de: https:// www.gcedclearinghouse.org/sites/default/files/ resources/232565s_0.pdf

UNESCO (2015). Reimaginar el futuro.
Innovación para todos los niños y niñas. Fondo de las Naciones Unidas para la Infancia (UNICEF). ISBN: 978-92-806-4782-2. Recuperado: https:// www.unicef.org/spanish/publications/files/ SOWC_2015_Summary_Spanish_Web.pdf

Weinberg. R. S., y Gould, D. S. (2014). Foundations of sport and exercise psychology. Champage, Unit State. Human Kinetics.

Número de cita totales/ Total references: 29 (100 $\%)$

Número de citas propias de la revista / Journal's own references: $1(30 \%)$ 